\title{
Functional Studies of Alzheimer's Disease Tau Protein
}

\author{
Qun Lu and John G. Wood \\ Department of Anatomy and Cell Biology, Emory University School of Medicine, Atlanta, Georgia 30322
}

\begin{abstract}
In vitro assays were used to monitor and compare the kinetic behavior of bovine tubulin polymerization enhanced by tau proteins isolated from Alzheimer's disease (AD) and nondemented (ND) age-matched control brains. Tau from AD cases induced slower polymerization and a steady state turbidity value approximately $50 \%$ of that stimulated by tau from control cases. Tau from the most severe AD case was least effective at promoting polymerization. Dark-field light microscopy of the control samples revealed abundant microtubule formation and many microtubule bundles. Microtubule assembly was observed in AD samples as well, but bundling was not obvious. These results were confirmed by negativestain electron microscopy. Morphological analysis showed that $A D$ tau-induced microtubules were longer than control microtubules. Furthermore, our initial results suggest that the reduction of $A D$ tau activity is correlated with neurofibrillary pathology in AD brains. Earlier reports indicated that $A D$ tau is modified by phosphorylation (Grundke-Iqbal et al., 1986; Wood et al., 1986; Iqbal et al., 1989; Brion et al., 1991 a,b; Lee et al., 1991). Our results support the hypothesis that tau modification compromises its function by altering its ability to nucleate and bundle microtubules.
\end{abstract}

IKey words: Alzheimer's disease, tau protein, microtubule assembly, functional alteration, kinetic assay, light and electron microscopy]

Microtubules are the fundamental organelle for fast axonal transport, which is essential for the renewal of axons and membranes in the nerve terminal. Defective microtubule assembly and stabilization in neurons, therefore, could lead to impaired axonal transport and abnormal synaptic transmission. The stability of microtubules in neurons can be achieved in a number of ways, including tubulin posttranslational modification and the regulation of microtubule-associated proteins (MAPs) of either the high-molecular-weight MAPs or the low-molecularweight tau proteins (for review, see Matus, 1988; Mitchison and Kirschner, 1988).

Tau proteins are a heat-stable family of developmentally regulated phosphoproteins that are generated by alternative splicing of a single gene (Drubin et al., 1984; Lee et al., 1988; Himmler, 1989; Kosik et al., 1989). In vitro, tau stimulates the assembly of microtubules at tubulin concentrations well below the critical

\footnotetext{
Received May 4, 1992; revised July 21, 1991; accepted July 23, 1992.

We thank Dr. L. Binder for providing mAb Tau-1, and Ms. J. Soria and Mr. R. Gopal for technical assistance. This work was supported by NIH Grants AG $06383, \mathrm{AG} 11123$, and NS 27847.

Correspondence should be addressed to J. G. Wood at the above address.

Copyright (C) 1993 Society for Neuroscience $0270-6474 / 93 / 130508-08 \$ 05.00 / 0$
}

concentration at which pure tubulin assembles (Weingarten et al., 1975; Cleveland et al., 1977). In cultured fibroblasts, which do not contain endogenous tau, microinjected tau can incorporate into microtubules and stabilize them against depolymerization conditions (Drubin and Kirschner, 1986; Lu and Wood, 1991b).

In brain, tau is largely localized in axons (Binder et al., 1985; Brion et al., 1988). However, in AD brain tau becomes an integral part of paired helical filaments (PHFs) in neurofibrillary tangles of neuronal cell bodies as well as dystrophic neurites associated with neuritic plaques (Brion et al., 1985; GrundkeIqbal et al., 1986, 1988; Kosik et al., 1986; Wood ct al., 1986). This dislocation is accompanied by abnormal phosphorylation (Grundke-Iqbal et al., 1986; Wood et al., 1986; Iqbal et al., 1989; Brion et al., 1991a; Lee et al., 1991). Recently, it was found that $\mathrm{A} 68$, a putative $\mathrm{AD}$-specific protein (Wolozin et al., 1986), is an abnormally phosphorylated tau and is the only component of a class of PHFs (Lee et al., 1991). The tubulins, the main building block of microtubules from AD brain, are functionally competent to reassemble and form microtubules in vitro, but microtubule assembly from $\mathrm{AD}$ brain homogenates is not observed (Iqbal et al., 1986). Thus, modification of tau may be responsible for the defective microtubule assembly, as phosphorylated bovine tau was found to be less efficient in promoting tubulin polymerization than dephosphorylated tau (Lindwall and Cole, 1984). Although it is reasonable to hypothesize that the alteration of tau distribution and chemistry contributes to the disruption of neuronal microtubule integrity and the formation of AD pathology, it has not been established whether AD tau, before it transforms into PHFs, is still functional. In this study, we used in vitro assays including kinetic analysis and subsequent dark-field light microscopy and electron microscopy to address the questions whether $\mathrm{AD}$ tau in soluble form is functionally competent to promote microtubule formation and whether the nicrotubules thus formed express distinct behavior and morphology when compared with that stimulated by ND tau. The results show that AD tau can stimulate microtubule assembly but with slower kinetics and different microtubule morphology, which supports the hypothesis that abnormal tau is involved in modification of the neuronal microtubule system and AD pathogenesis.

\section{Materials and Methods}

Protein purification. Bovine tubulin was isolated through temperaturedependent microtubule polymerization-depolymerization cycles and further purified by DEAE-Sephacel ion exchange column chromatography (Detrich, 1986). Tau was isolated using a modified method (Pollock and Wood, 1988) of perchloric acid extraction of heat-stable fractions (HSF) by Baudier et al. (1987). Fresh half AD or ND brains with average postmortem times of $7 \mathrm{hr}$ were homogenized in cold $20 \mathrm{~mm}$ 2 -[ $N$-morpholino]ethanesulfonic acid (MES) buffer, pH 6.85 with $2 \mathrm{~mm}$ 
ethylene glycol bis( $\beta$-aminoethyl) ether- $N, N, N^{\prime}, N^{\prime}$-tetra-acetic acid (EGTA), $1 \mathrm{~mm} \mathrm{MgSO}_{4}, 0.75 \mathrm{M} \mathrm{NaCl}$, and $1 \mathrm{~mm} \beta$-mercaptoethanol. Protease inhibitors leupeptin $(10 \mu \mathrm{g} / \mathrm{ml})$, pepstatin $\mathrm{A}(10 \mu \mathrm{g} / \mathrm{ml})$, aprotinin $(125 \mathrm{KIU} / \mathrm{ml} ; \mathrm{KIU}=$ Kallikrein inhibitory unit), and $2 \mathrm{~mm}$ phenylmethylsulfonyl fluoride (PMSF) were added fresh to the buffer before homogenization. The $75,000 \times g$ supernatant of brain homogenates was boiled at $95^{\circ} \mathrm{C}$ for $5 \mathrm{~min}$ in the same buffer with $0.75 \mathrm{M} \mathrm{NaCl}$ and 2 mM DL-dithiothreitol (DTT) to generate HSF. Proteins other than tau were precipitated by perchloric acid treatment and removed by centrifugation. Tau was also separated from high-molecular-weight microtubule-associated protein MAP-2 by molecular sieve chromatography of the HSF on a Bio-Gel A 1.5 M column. Tau protein used for kinetic experiments was concentrated using an ultrafiltration unit (Amicon Corp., Danvers, MA) to $3-5 \mathrm{mg} / \mathrm{ml}$ and centrifuged at $45,000 \times g$ for $20 \mathrm{~min}$ to remove aggregates before experiments. Protein concentrations were determined using a Bio-Rad protein assay based on the Bradford method (1976). Western blot analysis was performed as described previously (Wood et al., 1986). Quantitative analysis of the bands and the ratio of tau to tau fragments was performed using a Bio-Rad 620 video densitometer.

Pathology. Hippocampal slices from AD and ND brains were fixed in fresh PLP (paraformaldehyde, lysine, sodium periodate, and $\mathrm{NaCl}$ ) mixture of McLean and Nakane (1974). Vibratome sections, $50 \mu \mathrm{m}$, of hippocampus were stained using a modified Sevier-Munger silver impregnation method (Sevier and Munger, 1965). The occurrences of tangles and plaques were counted primarily in CA1 and CA4 subdivision of hippocampi at $200 \times$ under the Zeiss Axiovert 35 light microscope. The densities of tangles and plaques were determined by averaging the data from total area of $2.1-10.8 \mathrm{~mm}^{2}$ with statistical analysis performed using CRICKET GRAPH software on the Apple Macintosh IIci computer.

Kinetic analysis. DEAE-Sephacel tubulin was mixed with AD or ND tau at $4{ }^{\circ} \mathrm{C}$, and polymerization was initiated in $0.1 \mathrm{M}$ piperazine- $N, N^{\prime}-$ bis[2-ethanesulfonic acid] (PIPES) with $2 \mathrm{~mm}$ EGTA and $1 \mathrm{~mm} \mathrm{MgSO}$, $\mathrm{pH} 6.72$, by adding $1 \mathrm{~mm}$ guanosine $5^{\prime}$-triphosphate (GTP) and raising the temperature to $37^{\circ} \mathrm{C}$. The kinetic behavior was monitored by recording turbidity changes at $350 \mathrm{~nm}$ o.d. using a Beckman DU-64 UV spectrophotometer with a Kinetics Soft-Pac@ module and temperature controller.

Light and electron microscopy. After the kinetic measurements were completed, aliquots were taken from cuvettes for dark-field light microscopic examination. In addition, $5 \mu \mathrm{l}$ aliquots were dropped onto Formvar-coated grids and negatively stained with $2 \%$ uranyl acetate for electron microscopy. Some samples were fixed in $0.2 \%$ glutaraldehyde prior to electron microscopic (EM) examination. For thin-section electron microscopy, aliquots were centrifuged at $45,000 \times g$ for $30 \mathrm{~min}$ at $32^{\circ} \mathrm{C}$ to pellet microtubules. These samples were then fixed in $0.2 \%$ glutaraldehyde, postfixed in $1 \% \mathrm{OsO}_{4}$, and embedded for thin sectioning. Sections were examined and photographed using a Jeol 100 CX II transmission electron microscope.

Microtubule length determination. Microtubule numbers and lengths were determined first using thin-section electron microscopy. The ratio of long microtubules versus short microtubules (LMT/SMT) was determined on photographic prints. The numbers shown are averaged values \pm SEM. Long microtubules were arbitrarily defined as microtubules longer than $0.5 \mu \mathrm{m}$. In more recent studies, to obtain more global views of the microtubule population, microtubule lengths were measured on the photographs taken under dark-field light microscopy. In this case, single microtubules were counted and thick bundles of microtubules were omitted because it was not possible to determine the number and length of individual microtubules in the bundles. A microtubule length distribution was generated and statistical analysis performed using CRICKET GRAPH and CANVAS@ software on the Apple Macintosh II ci computer.

\section{Results}

\section{Differential kinetic activity of $A D$ and ND tau to induce microtubule assembly}

To study whether AD tau is capable of stimulating microtubule assembly, it is necessary to use purified tubulin and tau in the assembly assays. Fresh bovine brain microtubules were obtained through temperature-dependent polymerization-depolymerization cycles, and tubulin was further purified by DEAESephacel ion exchange column chromatography. Figure 1 shows

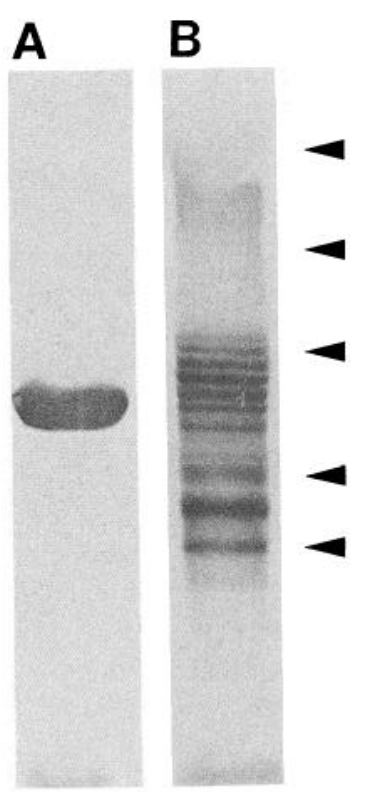

Figure 1. Western Blots of the proteins isolated for in vitro assays. A, Bovine tubulin immunostained with monoclonal anti- $\alpha$ tubulin DM1A; $B$, isolated human tau immunoblotted with $\mathrm{mAb}$ Tau-1. Gel lanes were loaded with $4.2 \mu \mathrm{g}$ total protein. Molecular weight standards are indicated on the right (arrowheads); from top to bottom: $205 \mathrm{kDa}, 116 \mathrm{kDa}$, $66 \mathrm{kDa}, 45 \mathrm{kDa}$, and $36 \mathrm{kDa}$.

Western blots of typical samples of bovine tubulin and human tau used for kinetic analysis. The bovine tubulin was devoid of microtubule-associated proteins as judged by Coomassie bluestained gels (data not shown). AD and ND tau were isolated based on the heat-stable and perchloric acid-soluble properties of tau proteins. Human tau proteins isolated by our method contained the major isoforms at 55-70 $\mathrm{kDa} \mathrm{MW}$ with some degradation products. These bands were reactive with Tau-1 monoclonal anti-tau (a gift generously provided by Dr. L. Binder at the University of Alabama, Birmingham) and polyclonal antitau (Sigma, St. Louis, MO). MAP-2 was not detected on Coomassie blue-stained gels or on Western blots (data not shown). Densitometry was employed to compare the amount of tau isoforms and their proteolytic fragments among cases and we found no significant differences by statistical analysis ( $t$ test, $p$ $>0.01, n=4)$.

Above the critical concentration, tubulin can self-polymerize in vitro in an appropriate buffer with addition of GTP and raising the temperature to $37^{\circ} \mathrm{C}$ (Weingarten et al., 1975). When microtubules are formed, they scatter light and the solution becomes viscous. The light scattering can be measured quantitatively by recording the turbidity changes at $350 \mathrm{~nm}$ o.d. When ND tau and AD tau are mixed with DEAE-Sephacel tubulin and polymerization is initiated as described above, a microtubule assembly kinetics recording is obtained (Fig. $2 A$ ). This figure compares one case pair of AD and ND tau for their ability to stimulate microtubule assembly, and it shows that $\mathrm{AD}$ tau can induce tubulin polymerization but at a slower rate and less effectively than ND tau. In fact, the level of polymerization at steady state for the AD sample was only $68 \%$ of that for the ND control. Figure $2 B$ summarizes kinetic experiments testing a total of eight cases (five AD samples and three ND controls) for their tau activities. Again, AD tau appeared to be able to induce tubulin to polymerize when compared with tubulin self- 
Figure 2. Turbidimetric assay illustrating the kinetic behavior of bovine tubulin polymerization induced by $\mathrm{AD}$ tau and age-matched ND control tau. $A$, One pair of AD and ND tau was tested for their activity to promote microtubule assembly monitored by turbidity changes at o.d. $350 \mathrm{~nm}$. Squares, ND tau activity; triangles, AD tau activity. [tubulin], $2.99 \mathrm{mg} / \mathrm{ml}$; [tau], 0.2 $\mathrm{mg} / \mathrm{ml}$. $B$, Summary of the tau activity for eight $\mathrm{AD}$ and ND cases. Open squares, ND tau activity and each point is an average value from three cases; solid squares, AD tau activity and each point is an average value from five cases; triangles, tubulin self assembly and each point represents two independent experiments.
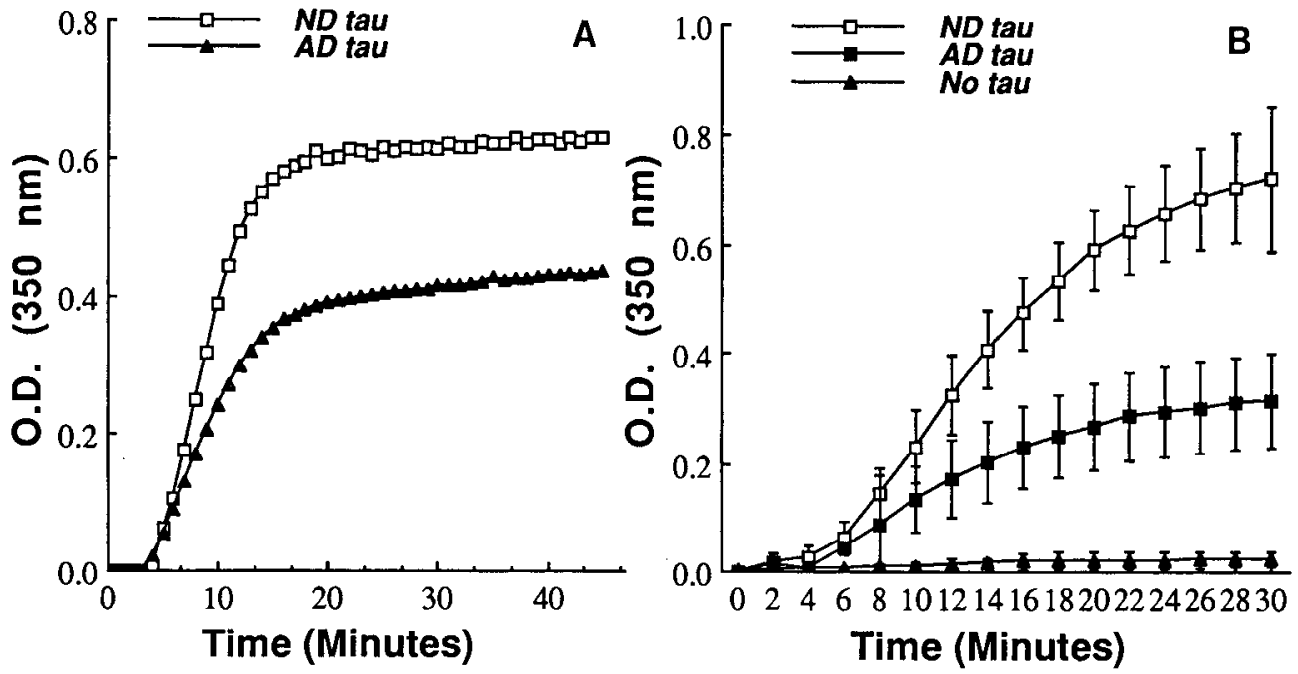

polymerization. However, their activities were greatly reduced to an average value for all cases of $50.57 \%$ of that for ND controls. These results suggested that the AD tau ability to induce microtubule assembly in vitro was altered quantitatively but not qualitatively.

\section{Microtubule morphology and behavior}

Although recording of turbidity changes enables us to measure quantitatively and therefore differentiate AD tau and ND tau for their abilities to induce tubulin polymerization, alternative assays are required to demonstrate microtubule formation and structure. In earlier studies, microtubules were pelleted by centrifugation after the kinetic measurement was completed and then prepared for thin-section electron microscopy. Figure 3 shows that both ND and AD tau were able to induce microtubule formation. The ultrastructure of the individual microtubules from ND control and AD samples appeared similar. However, large numbers of microtubules in ND controls were of relatively short size (Fig. 3B), while self-assembled microtubules were significantly longer (Fig. $3 A$ ). When the ratio of long microtubules to short microtubules (LMT:SMT) was correlated with the amplitude of assembly (represented as the percentage change of turbidity at the steady state of tubulin polymerization; for ND tau, the activity was assigned as $100 \%$ turbidity change in each paired experiment), the ND tau control had a very low LMT:SMT ratio (Fig. 4 $A$ ). Interestingly, $\mathrm{AD}$ tau-induced microtubule populations showed an LMT:SMT between ND controls and self-assembly (Figs. 3C, 4A), which agrees with the kinetic data. This observation is consistent with other work showing that under steady state conditions of assembly, MAPdepleted microtubules were longer than MAP-rich microtubules (Farrell et al., 1987).

To obtain a more global view of microtubule populations, dark-field light microscopy was employed to compare the length distribution of microtubules assembled in the presence of AD or ND tau. When aliquots taken from the cuvettes where tubulin polymerization had reached steady state were examined, numerous microtubules were observed in both ND controls and AD samples (Fig. 5). However, calculation of microtubule length distribution indicated that ND controls had more, but shorter, microtubules than AD samples (Fig. 6). Five cases in two paired experiments were studied for microtubule length distribution, and the mean microtubule lengths were $2.04 \pm 0.17 \mu \mathrm{m}$ for ND microtubules versus $3.58 \pm 0.41 \mu \mathrm{m}$ for AD microtubules. Thus, microtubule length analysis from both EM and dark-field light microscopic data demonstrated a correlation of higher amplitude of assembly to shorter microtubule lengths (Fig. 4). A reasonable interpretation of these results is that when there are finite tubulin molecules available for polymerization, a greater number of microtubule seeds are formed and stabilized in the presence of more assembly-competent ND tau molecules so that at steady state there are more microtubules of shorter size.

Dark-field light microscopy revealed another interesting difference between AD and ND tau-induced microtubule formation. Extensive bundling or grouping of microtubules was observed in ND controls (Fig. 5A,C), but this was rarely seen in AD samples (Fig. $5 B, D$ ). Negative-stain electron microscopy confirmed the bundling in ND controls, and also confirmed minimal microtubule bundling in $\mathrm{AD}$ samples (Fig. $7 A, B$ ).

\section{Tau activity in vitro is correlated with Alzheimer pathology}

We then examined the relationship between the reduction of AD tau activity and the severity of the disease. The presence of neurofibrillary tangles and neuritic plaques in the hippocampus, the amygdala, and frontal and temporal cortices is the hallmark pathology in AD and is correlated with the degree of dementia (Rewcastle, 1991). We selected hippocampus to measure tangle and plaque densities in $7-10$ areas each of $\sim 0.3$ $\mathrm{mm}^{2}$. The average value was compared against the amplitude of microtubule assembly obtained as described earlier. Table 1 shows the correlation of tangle and plaque density with the amplitude of microtubule assembly. The ability of tau protein to stimulate microtubule assembly was correlated with the tangle density such that a greater number of tangles was reflected in lower tau activity.

\section{Discussion}

In this article we have demonstrated that AD and ND tau exhibit differential abilities to stimulate microtubule assembly, and ND tau-induced microtubules are both shorter and more bundled than those induced by AD tau. Furthermore, initial comparisons suggest that the reduction of AD tau activity is correlated with an increase in neurofibrillary tangle density in AD brain hippocampus. The results predict consequences for microtubule 

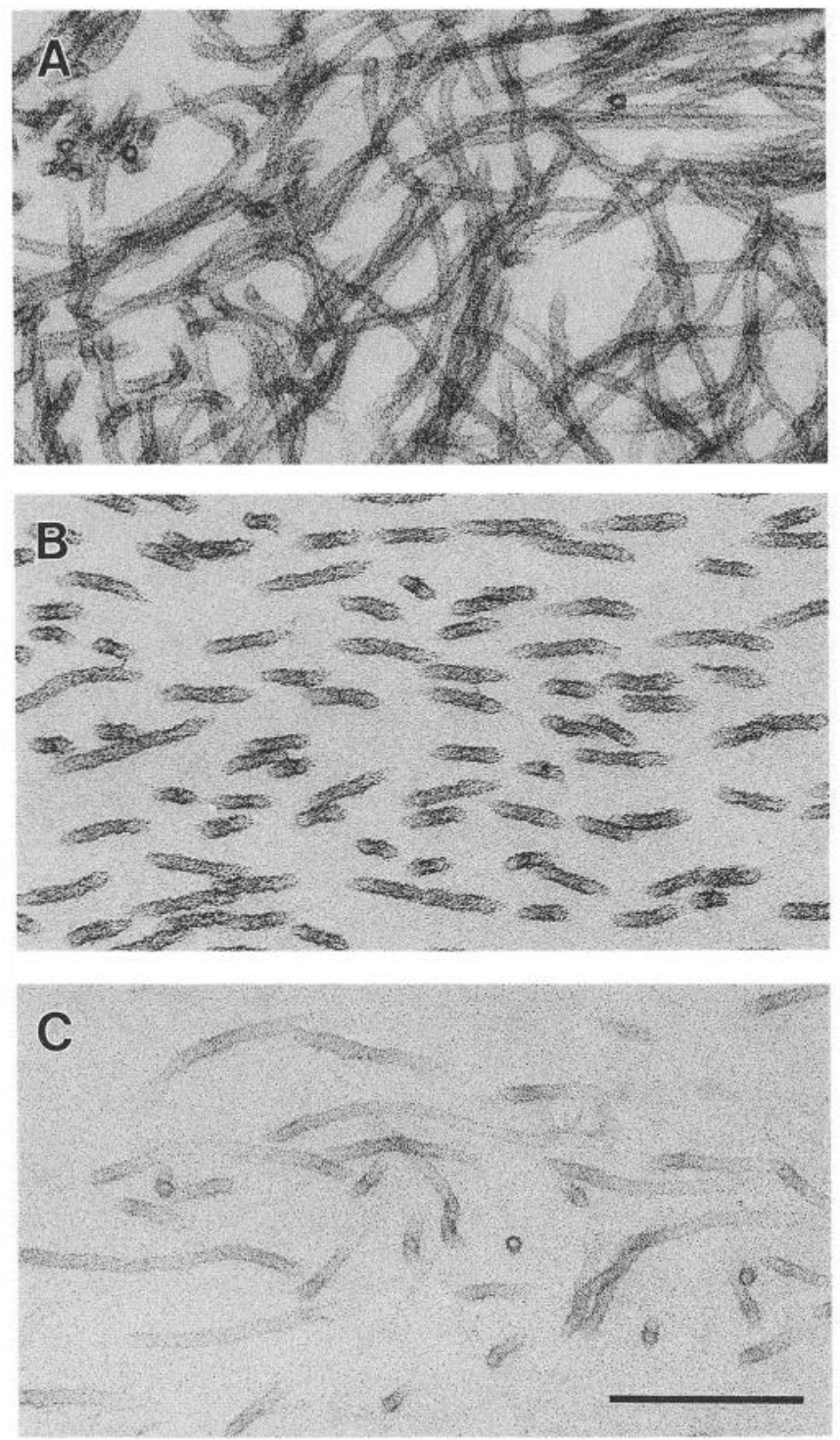

Figure 3. Thin-section electron micrographs of the assembled microtubules. $A$, Self-polymerized microtubules. $B$, ND tau-induced microtubule formation. $C$, AD tau-induced microtubule formation. Self-polymerized microtubules are long. Note that the ultrastructure of ND and AD microtubules appeared similar; however, the majority of ND microtubules are of relatively short size while the AD microtubule fraction contains both long and short microtubules. Scale bar, $0.3 \mu \mathrm{m}$.

stability, axonal transport functions, and potential mechanisms for PHF formation in AD brain.

The assembly data show that the AD tau function of promoting microtubule assembly is compromised compared to ND tau. A likely consequence is that the microtubule system in disease-affected neurons would be defective. This prediction is supported by studies of Iqbal et al. (1986). They showed that microtubule reassembly from $\mathrm{AD}$ brain homogenates was not observed, whereas neurofilaments could be reassembled. They further showed that tubulins from AD brains were competent to form microtubules with the addition of DEAE-Dextran. Nieto et al. (1990) showed that HSFs, prepared from AD brains and incubated with purified porcine tubulin, generated protein aggregates and that microtubules were rarely observed. Nieto et al. (1991) further demonstrated that the binding to microtubules
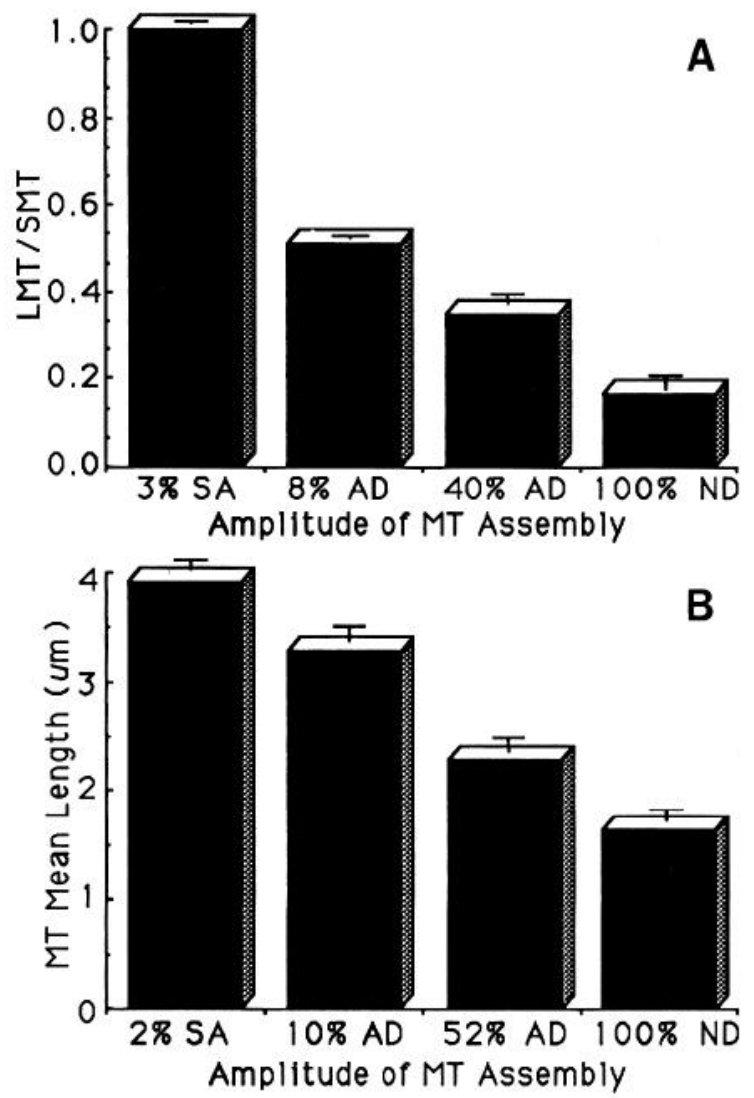

Figure 4. Histograms showing the relationship between microtubule (MT) length and amplitude of microtubule assembly. $A$, Microtubule length ratio correlated with amplitude of microtubule assembly. $L M T$ / $S M T$, the ratio of long microtubule number to short microtubule number. Long microtubules are arbitrarily defined as longer than $\sim 0.5 \mu \mathrm{m}$. The microtubule numbers and lengths were determined on electron micrograph prints. $B$, Mean of microtubule length correlated with amplitude of microtubule assembly. The microtubule lengths were measured on dark-field light micrograph prints. All the data shown here are averaged value \pm SEM. The amplitude of assembly is represented as the percentage change of turbidity at steady state of tubulin polymerization, and the amplitude of assembly for ND tau activity is assigned to $100 \%$ for each paired experiment. The LMT:SMT for self-assembled microtubules $(S A)$ is assigned as 1 .

of tau-related fragments isolated from PHFs was reduced. The prediction is further supported by the observations of Ellisman et al. (1987), who used three-dimensional reconstruction from serial sections of biopsy tissue to show a paucity of microtubules in the cytoplasm of tangle-bearing neurons. In the present study, we provide evidence that directly points to $A D$ tau as the cause of the defective microtubule system in AD brain. This conclusion is strengthened by our use of a defined in vitro system that used only purified bovine tubulin and AD or ND tau proteins.

The reduced ability of AD tau to stimulate microtubule assembly and the subsequent paucity of microtubules in affected neurons would have direct consequences for the cell. Axons require microtubule-based fast axonal transport for renewal of membrane components. The efficient operation of this system requires a relatively stable population of microtubules. The unstable population predicted from our results and those of other groups would directly and adversely affect axonal transport processes. Over time and with significant diminution in microtubule number, the neuron would reach a state of inability to maintain distal axonal processes. The cell would degenerate 

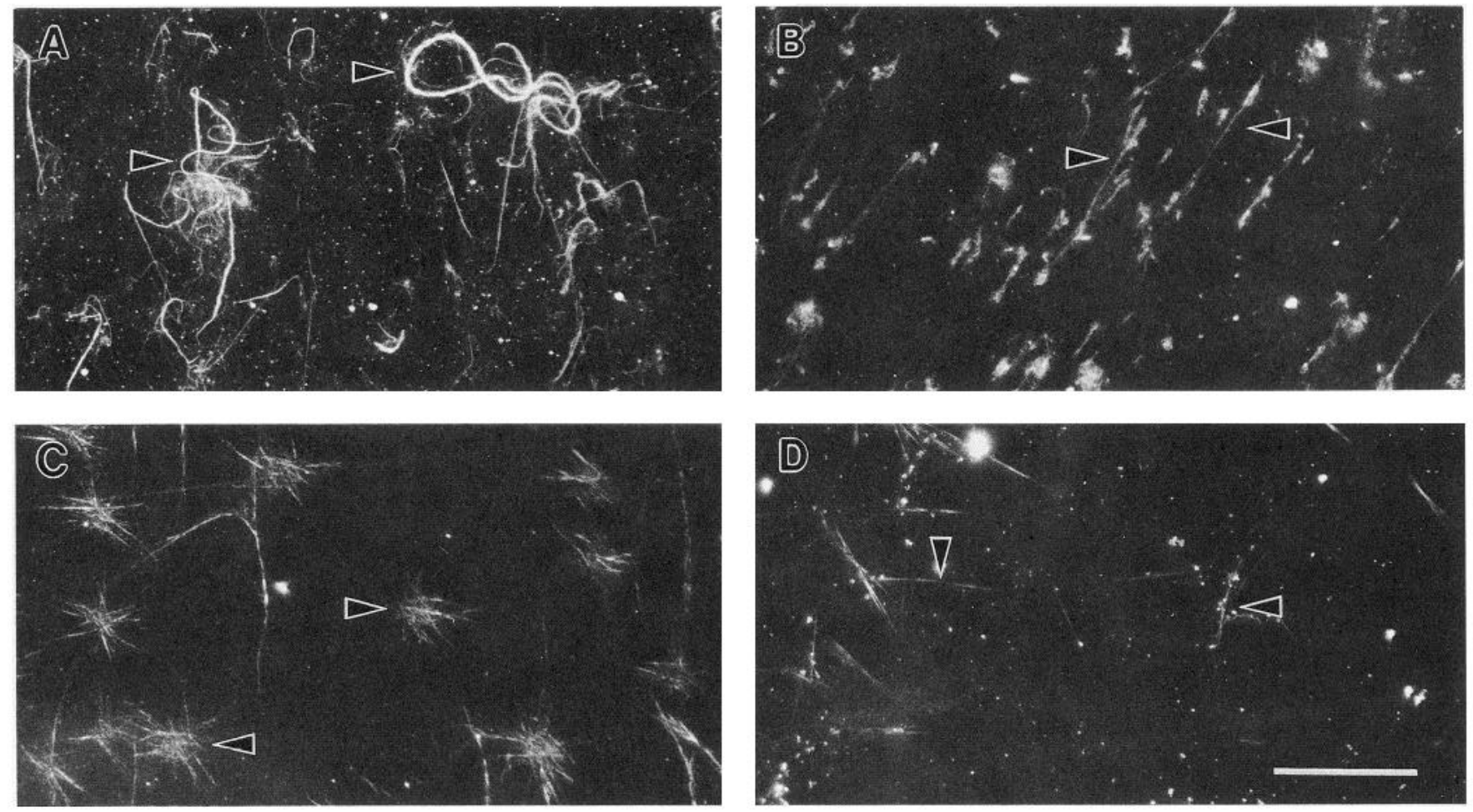

Figure 5. Assembled microtubule populations visualized by dark-field light microscopy. $A$ and $C$ show the morphology of assembled microtubule population induced by ND tau. Note that in $A$ there are both single microtubules and microtubule bundles (arrowheads). In $C$, there are many short microtubules grouped together (arrowheads) with some long microtubules. $B$ and $D$ show the morphology of assembled microtubules induced by AD tau. Note that in $B$ and $D$ there are many microtubules (arrowheads) but microtubule bundles similar to $A$ and $C$ are not seen. Scale bar, $10 \mu \mathrm{m}$.
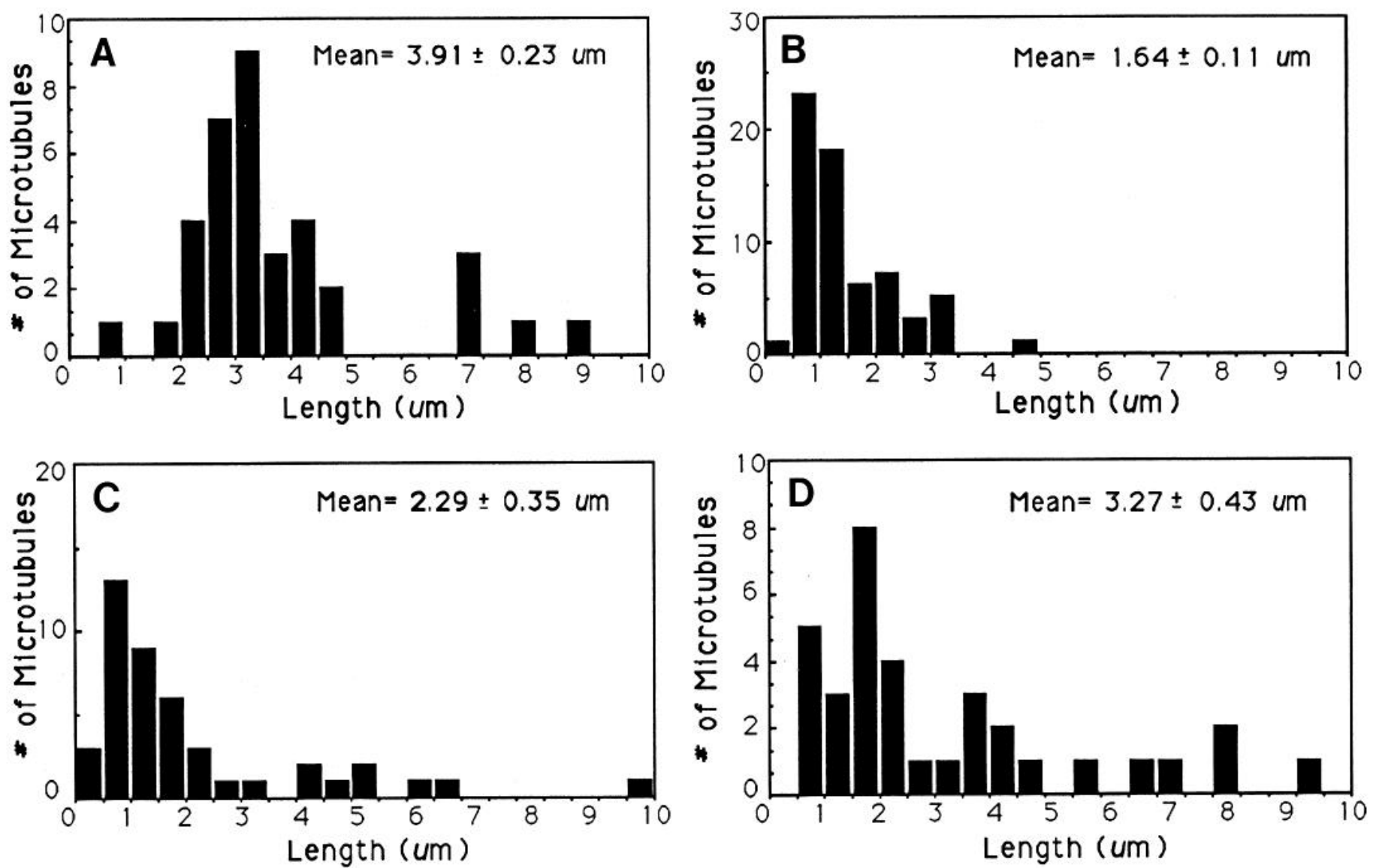

Figure 6. Histograms showing examples of length distribution of microtubules measured from dark-field light microscopy at steady states. A, Selfassembled microtubules. $B$, ND tau-induced microtubules. $C$ and $D, A D$ tau-induced microtubules. 


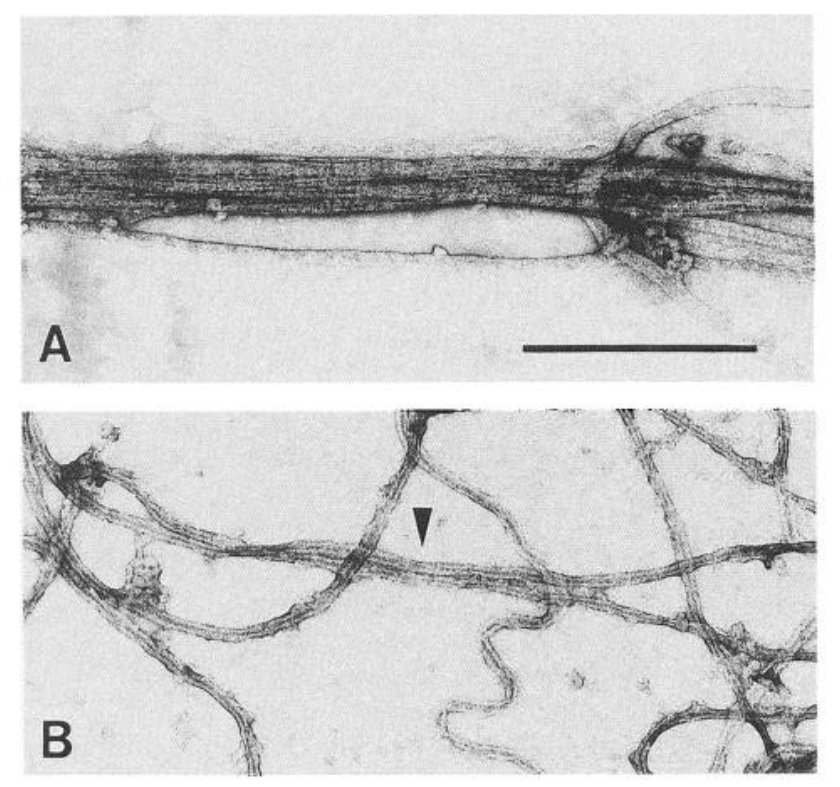

Figure 7. Ultrastructure of assembled microtubules visualized by electron microscopy with negative staining. $A$, ND tau-induced microtubules showing distinct bundling; $B, \mathrm{AD}$ tau-induced microtubules. Note that the microtubule bundling is not obvious although there are possible associations between adjacent microtubules (arrowhead). Scale bar, 0.5 $\mu \mathrm{m}$.

those processes, thus severing the functional connection of the disease-affected neuron to its ensemble of neurons forming a circuit involved in mediating cognitive function and behavior.

The observation that soluble tau not yet incorporated into soluble or insoluble PHFs has compromised ability to assemble microtubules has implications for AD pathogenesis. Neurofibrillary tangles from AD brains are highly insoluble even in strong detergents such as SDS. Greenberg and Davies (1990) isolated a distinct group of PHFs soluble in SDS buffer and sharing similarities with tau proteins. Lee et al. (1991) directly demonstrated that A68, a putative AD-specific protein (Wolozin et al., 1986), is a modified form of normal tau and is the major (or only) component of a group of SDS-soluble PHFs. At present, we do not know how normal tau, which is soluble in aqueous solution, transforms into SDS-insoluble PHFs, but the presence of several intermediates, including the tau reported in this article, suggests a sequence of tau modifications. However, Bramblett et al. (1992) suggested that soluble tau in AD brain may be transformed to A68 rapidly without involvement of an intermediate stage. We have previously demonstrated that the soluble tau isolated by our methods is modified by phosphorylation at the site recognized by the Tau- 1 antibody (Pollock and Wood, 1988). Lee et al. (1991) have shown that their SDSsoluble PHF tau is modified by phosphorylation on at least two acceptor sites. Thus, a plausible model for PHF formation would include a series of phosphorylation events at several aberrant acceptor sites (Grundke-Iqbal et al., 1986; Wood et al., 1986; Iqbal et al., 1989; Brion et al., 1991a; Kopke-Secundo et al., 1991; Lee et al., 1991). It may be of interest that the soluble tau we are studying is apparently an early modified form, yet is already deficient in its functional ability to assemble microtubules. This property likely affects microtubule function as elaborated above, but other offshoots are possible as well. One particularly meaningful possibility is that AD tau proteins, deprived
Table 1. Relationship between amplitude of microtubule assembly and Alzheimer cytoskeletal pathology

\begin{tabular}{llllll} 
Case & Sex/age & Tangles $^{a}$ & Plaques $^{a}$ & $\begin{array}{l}\text { Ampli- } \\
\text { tude }^{b} \\
\text { (MT as- }_{\text {sembly) }}\end{array}$ & $\begin{array}{l}\text { Clinical } \\
\text { diagnosis }^{2}\end{array}$ \\
\hline 1 & F/55 & - & - & 100 & ND \\
2 & M/75 & - & - & 100 & ND \\
3 & M/92 & - & - & 100 & ND \\
4 & F/77 & $6 \pm 0.80$ & $9 \pm 0.77$ & 68 & AD \\
5 & F/85 & $4 \pm 2.02$ & $37 \pm 8.13$ & 52 & AD \\
6 & M/64 & $59 \pm 0.85$ & $22 \pm 0.90$ & 8 & AD
\end{tabular}

$\bar{a}$ AD cytoskeletal pathology: shown as number of tangles or plaques in hippocampi per square millimeter $\pm \mathrm{SEM} ;-$, very few/not detected.

${ }^{b}$ Amplitude of microtubule (MT) assembly is represented as percentage change of turbidity at the steady state of microtubule assembly; amplitude of MT assembly for ND tau activity is assigned as $100 \%$.

of their normal interaction with microtubules, may accumulate as free proteins where they would be available to self-associate as polymers. Over time and with additional phosphorylation, these polymers could progressively alter into the final SDSinsoluble neurofibrillary tangle. It should be noted that the functional deficit in AD tau may be more pronounced than we have demonstrated. Although we make every effort to ensure that our starting brain material is selected to allow enrichment in tau from regions of abundant pathology, our samples undoubtedly contain some normal tau from nonaffected neurons. This would shift the kinetic data toward that observed for the control samples, so that the significant decrease in functional activity we observe may actually be greater for the modified tau.

We used dark-field light microscopy and EM to demonstrate that microtubules assembled in the presence of $\mathrm{AD}$ tau were longer than self-assembled microtubules but shorter than microtubules assembled by ND tau. This supports the hypothesis that $\mathrm{AD}$ tau function is altered since, at steady state conditions, MAP-deficient microtubules are generally longer than MAPrich microtubules (Farrell et al., 1987).

We also presented evidence that AD tau may be less efficient at bundling microtubules than ND tau. In addition to its role in microtubule assembly in vitro (Weingarten et al., 1975; Cleveland et al., 1977), tau can bind to and stabilize microtubules in cultured RAT-1 cells (Drubin and Kirschner, 1986) and CHO cells (Lu and Wood, 1991b). Transfected cells overexpressing tau exhibit distinct bundles of microtubules that are highly resistant to microtubule depolymerizing drug treatments (Kanai et al., 1989; Lewis et al., 1989; Bass et al., 1991; Knops et al., 1991). The functional consequences of microtubule bundling induced by tau is presently unclear, but it could play a role in microtubule stabilization in axons. This is particularly possible at the initial segment where microtubules are tightly packed (Peters et al., 1991). We are currently using microinjection protocols to investigate the properties of AD and ND tau with respect to microtubule bundling in living cells. Regardless of the ultimate significance of microtubule bundling to neuronal function, the present results offer one more property whereby $\mathrm{AD}$ tau is altered in its functional capacity.

Our initial results suggest that the ability of tau to stimulate microtubule assembly is negatively correlated with the degree 
of cytoskcletal pathology, particularly the neurofibrillary tangle. Although this is clearly a satisfying trend, additional cases will be required to illustrate the detailed specifics of this correlation.

In summary, we have shown that a soluble form of $\mathrm{AD}$ tau is less efficient than ND tau at promoting microtubule assembly. This AD tau is in an early stage of modification that is most likely an aberrant phosphorylation event at one or more sites. A predicted consequence supported by a vailable evidence is that axonal microtubules would be unstable. This in turn would adversely affect fast axonal transport processes critical to survival of the neuron. Consistent with the kinetic data, microtubule morphology and behavior are different in the presence of $\mathrm{AD}$ compared to ND tau. An additional functional consequence possible from these observations is that tau not competent to promote assembly may exist as a free monomer that could self-associate to form polymer. In a stepwise fashion accompanied by additional phosphorylation events, the polymer could be converted into the SDS-insoluble neurofibrillary tangle. Experiments to address the latter possibility are underway.

\section{References}

Bass PW, Pienkowski TP, Kosik KS (1991) Processes induced by tau expression in Sf 9 cells have an axon-like microtubule organization. J Cell Biol 115:342a.

Baudier J, Lee S-H, Cole RD (1987) Separation of the different microtubule-associated tau protein species from bovine brain and their mode II phosphorylation by $\mathrm{Ca}^{11} /$ phospholipid-dependent protein kinase C. J Biol Chem 262:17584-17590.

Binder LI, Frankfurter A, Rebhun LI (1985) The distribution of tau in the mammalian central nervous system. J Cell Biol 101:13711378.

Bradford MM (1976) A rapid and sensitive method for the quantitation of microgram quantities of protein utilizing the principle of protein-dye binding. Anal Biochem 72:248-254.

Bramblett GT, Trojanowski JQ, I ee VM-Y (1992) Regions with abundant neurofibrillary pathology in human brain exhibit a selective reduction in levels of binding-competent $\tau$ and accumulation of abnormal $\tau$-isoforms ( $\Lambda 68$ proteins). Lab Invest $66: 212-222$.

Brion JP, Passareiro JP, Nunez J, Flament-Durand J (1985) Mise en evidence immunologique de la proteine tau au niveau des lesions de degenerescence neurofibrillaire de la maladie d'Alzheimer. Arch Biol (Bruxells) 95:229-235.

Brion JP, Guilleminot J, Couchi D, Flament-Durand J, Nunez J (1988) Both adult and juvenile tau microtubule-associated proteins are axon specific in the developing and adult rat cerebellum. Neuroscience 25: 139-146.

Brion JP, Hanger DP, Bruce MT, Couck A, Flament-Durand J, Anderton BH (1991a) Tau in Alzheimer neurofibrillary tangles: $\mathrm{N}$ - and C-terminal regions are differentially associated with paired helical filaments and the location of a putative abnormal phosphorylation site. Biochem J 273:127-133.

Brion JP, Hanger DP, Couck A, Anderton BH (1991b) A68 proteins in Alzheimer's disease are composed of several tau isoforms in a phosphorylated state which affects their electrophoretic mobilities. Biochem J 279:831-836.

Cleveland DW, Hwo S-Y, Kirschner MW (1977) Purification of tau, a microtubule-associated protein that induces assembly of microtubules from purified tubulin. J Mol Biol 116:207-225.

Detrich III HW (1986) Isolation of sea urchin egg tubulin. Methods Enzymol 134:128-138.

Drubin D, Kirschner MW (1986) Tau function in living cells. J Cell Biol 103:2739-2746.

Drubin D, Kirschner MW, Feinstein S (1984) Microtubule-associated tau protein induction by nerve growth factor during neurite outgrowth in PC 12 cells. In: Molecular biology of the cytoskeleton (Borisy G, Clevcland D, Murphy D, eds), pp 343-355. Cold Spring Harbor, NY: Cold Spring Harbor Laboratories.

Ellisman M, Raganathan R, Deerinck T, et al. (1987) Neurofibrillary cytoskeleton and endomembrane system organization in Alzheimer's disease. Adv Behav Biol 34:61-73.
Farrell KW, Jordan MA, Miller HP, Wilson L (1987) Phase dynamics at microtubule ends: the coexistence of microtubule length changes and treadmilling. J Cell Biol 104:1035-1046.

Greenberg S, Davies P (1990) A preparation of Alzheimer paired helical filaments that displays distinct tau proteins by polyacrylamide gel electrophoresis. Proc Natl Acad Sci USA 87:5827-5831.

Grundke-lqbal 1, lqbal K, Tung YCH, Quinlan M, Wisniewski HM, Binder LI (1986) Abnormal phosphorylation of the microtubuleassociated protein tau in Alzheimer cytoskeletal pathology. Proc Natl Acad Sci USA 83:4913-4917.

Grundke-Iqbal I, Vorbrodt AW, Iqbal K, Tung YCH, Wang GP, Wisniewski HM (1988) Microtubule-associated polypeptides tau are altered in Alzheimer paired helical filaments. Mol Brain Res 4:4352 .

Himmler $\Lambda$ (1989) Structure of the bovine tau gene: alternative spliced transcripts generate a protein family. Mol Cell Biol 9:1389-1396.

Iqbal K, Grundke-Iqbal I, Zaidi T, Merz PA, Wen GY, Shaikh SS, Wisniewski HM (1986) Defective brain microtubule assembly in Alzheimer's disease. Lancet Aug 23:421-426.

Iqbal K, Grundke-Iqbal I, Smith AJ, George L, Tung YCH, Zaidi T (1989) Identification and localization of a $\tau$ peptide to paired helical filaments of Alzheimer disease. Proc Natl Acad Sci USA 86:56465650.

Kanai $Y$, Takemura R, Oshima T, Mori H, Ihara Y, Yanagissawa M, Masaki T, Hirokawa N (1989) Expression of multiple tau isoforms and microtubule bundle formation in fibroblasts transfected with a single tau cDNA. J Cell Biol 109:1173-1184.

Knops J, Kosik KS, Lee G, Pardee JD, Cohen-Gould L, McConlogue L (1991) Overexpression of tau in a nonneuronal cell induces long cellular processes. J Cell Biol 114:725-733.

Kopke-Secundo E, Grundke-Iqbal I, Iqbal K (1991) Abnormal phosphorylation of tau is one of the earliest events in Alzheimer neurofibrillary pathology. Soc Neurosci Abstr 17:1070.

Kosik KS, Joachim CL, Selkoe DJ (1986) Microtubule-associated protein tau is a major antigenic component of paired helical filaments in Alzheimer's disease. Proc Natl Acad Sci USA 83:4044-4048.

Kosik KS, Orecchio LD, Bakalis S, Neve RL (1989) Developmentally regulated expression of specific tau sequences. Neuron 2:1389-1397.

Lee G, Cowan N, Kirschner M (1988) The primary structure and heterogeneity of tau protein from mouse brain. Science 239:285-288.

Lee VM-Y, Balin BJ, Otvos Jr L, Trojanowski JQ (1991) A 68: a major subunit of paired helical filaments and derivatized forms of normal tau. Science 251:675-678.

Lewis SA, Ivanov IE, Lee GH, Cowan NJ (1989) Organization of microtubules in dendrites and axons is determined by a short hydrophobic zipper in microtubule-associated proteins MAP2 and tau. Nature 342:498-505.

Lindwall G, Cole RD (1984) Phosphorylation affects the ability of tau protein to promote microtubule assembly. J Biol Chem 259:53015305.

Lu Q, Wood JG (1991) Properties of fluorescently derivatized bovine tau protein. J Cell Biol 115:384a.

Matus A (1988) Microtubule-associated proteins: their potential role in determining neuronal morphology. Annu Rev Neurosci 11:29-44.

McLean IW, Nakane PK (1974) Periodate-lysine-paraformaldehyde fixative. A new fixative for immunoelectron microscopy. J Histochem Cytochem 22:1077-1083.

Mitchison T, Kirschner MW (1988) Cytoskeletal dynamics and nerve growth. Neuron 1:761-772.

Nieto A, Montejo de Garcini E, Correas I, Avila J (1990) Characterization of tau protein present in microtubules and paired helical filaments of Alzheimer's disease patient's brain. Neuroscience 37:163170 .

Nieto A, Correas I, Lopez-Otin C, Avila J (1991) Tau-related protein present in paired helical filaments has a decreased tubulin binding capacity as compared with microtubule-associated protein tau. Biochim Biophys Acta 1096:197-204.

Peters A, Palay S, Webster HD (1991) The fine structure of the nervous system, 3d ed. New York: Oxford UP.

Pollock NJ, Wood JG (1988) Differential sensitivity of the microtubule-associated protein, tau, in Alzheimer's disease tissue to formalin fixation. J Histochem Cytochem 36:1117-1121.

Rewcastle NB (1991) Degenerative disease of the central nervous system. In: Textbook of neuropathology (Davis RL, Robertson DM, eds), pp 904-918. Baltimore: Williams and Wilkins. 
Sevier AC, Munger BL (1965) A silver method for paraffin sections of neural tissue. J Neuropathol Exp Neurol 24:130-135.

Weingarten MD, Lockwood AH, Hwo SY, Kirschner MW (1975) A protein factor essential for microtubule assembly. Proc Natl Acad Sci USA 72:1858-1862.

Wolozin BL, Pruchnicki A, Dickson DW, Davies P (1986) A neuronal antigen in the brains of Alzheimer patients. Science 232:648-650.
Wood JG, Mirra SS, Pollock NJ, Binder LI (1986) Neurofibrillary tangles of Alzheimer disease share antigenic determinants with the axonal microtubule associated protein, tau. Proc Natl Acad Sci USA 83:4040-4043. 